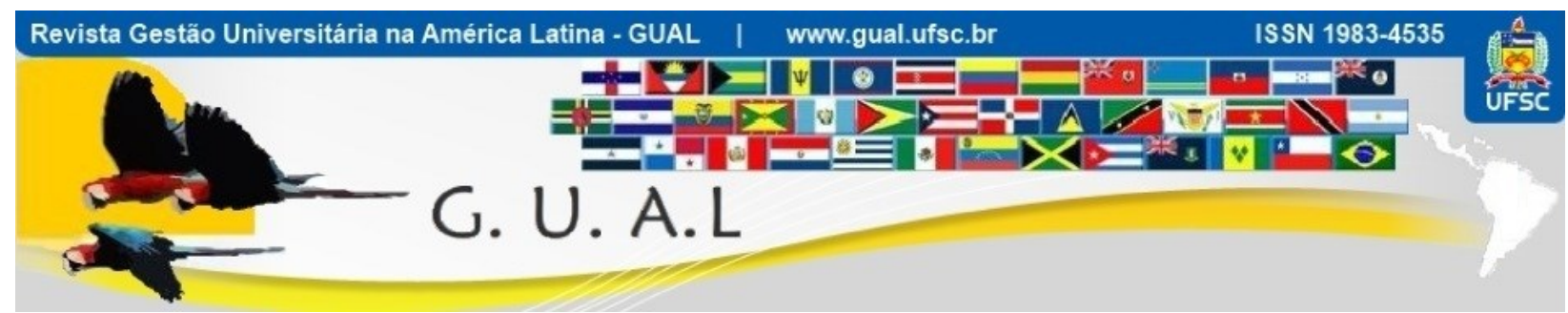

DOI: http://dx.doi.org/10.5007/1983-4535.2016v9n1p82

\title{
GESTÃO DA PRODUÇÃO CIENTÍFICA DE UMA REDE DE ENSINO SUPERIOR BRASILEIRA: ESTUDO DE CASO
}

\author{
SCIENTIFIC PRODUCTION MANAGEMENT OF A BRAZILIAN HIGHER \\ EDUCATION NETWORK: CASE STUDY
}

\author{
Luciano Vicente Medeiros, Pós-Doutor \\ Universidade Estácio de Sá - UNESA \\ luciano.medeiros@estacio.br \\ Valéria Goytacazes Coutinho, Especialista \\ Universidade Estácio de Sá - UNESA \\ valeria.coutinho@estacio.br \\ Renato Paes de Souza, Especialista \\ Universidade Estácio de Sá - UNESA \\ renato.souza@estacio.br
}

Recebido em 28/abril/2014

Aprovado em 29/setembro/2015

Sistema de Avaliação: Double Blind Review

Esta obra está sob uma Licença Creative Commons Atribuição-Uso. 


\title{
RESUMO
}

O presente trabalho apresenta procedimento utilizado para acompanhamento da atividade de pesquisa em uma rede de Educação Superior com 35 Instituições operando em 82 campi e 49 municípios. A metodologia utilizada recolhe, sistematizadamente, informações a respeito da produção científica dos docentes. $\mathrm{O}$ indicador, Índice de Produtividade em Pesquisa (IPP), pondera o quantitativo de selecionados, tipos de publicação científica e orientações de alunos em um espaço de tempo. A extensão deste conceito para Curso e Instituição de Ensino se obtém a partir da média de uma parcela predeterminada dos mais elevados IPPs. O percentual de docentes com IPP diferente de zero, reflete o grau de disseminação da pesquisa, definido como Nível de Envolvimento em Pesquisa (NEP). Este procedimento localiza níveis de excelência em pesquisa, identifica necessidades de correções de rumo e otimiza recursos destinados à pesquisa. A aplicação desta metodologia consecutivamente por cinco anos, sua incorporação às práticas de gestão e a periódica divulgação de resultados se revelaram como um eficiente mecanismo para o desenvolvimento de seus projetos acadêmicos. A atividade de pesquisa, desde que conduzida em sintonia com o ensino, possui elevado potencial de impactar positivamente o processo educacional. Este trabalho apresenta uma contribuição para a gestão da atividade de pesquisa.

Palavras Chave: Administração da Educação Superior. Gestão do conhecimento. Nexo Ensino e Pesquisa.

\begin{abstract}
The paper presents a procedure to manage research activity of the academic staff a of 35 higher education institution network having 87 campi along 49 municipalities. The proposed methodology makes a systematic database search of the scientific production of its academic staff. A Research Productivity Index (IPP), which makes a weighted balanced of selected types of scientific publication and student supervision, was conceived. This rational was extended to evaluate Research Productivity of Undergraduate Programs and Institutions, which is the average IPP of a predetermined fraction of their most productive academics. The percentage of academics with non-null IPP was defined as Research Engagement Ratio (NEP), which reveals as the research atmosphere is disseminated among its faculty. This praxis made it possible to identify research excellence, the necessary corrections, as well as to optimize available research funding. The use of this methodology for five consecutive years, its embrace to the institutional management practices followed by reports disclosure, has proven to be an efficient mechanism to improve research capability. Whenever research activities are developed in tune with teaching, they evolve into an important ingredient to maximize results to the educational system. The paper presents a contribution to research management practices in higher education institutions.
\end{abstract}

Keywords: Higher Education Administration. Knowledge Management. Teaching-Research Nexus. 


\section{INTRODUÇÃO}

A relação entre intensidade da atividade de pesquisa é muito debatida no meio acadêmico por diversos autores advogando em campos opostos, como relatado, entre outros, por Hattie and Marsh (2004), Nunes (2012), Cesar et al (2014), Geschwind e Brostrom (2015). As discussões se destinam a associar a qualidade das aulas do docente com o seu envolvimento em pesquisa. É, no entanto, reconhecidamente positiva a atitude dos docentes que se dedicam a trazer para sala de aula relevantes experiências que não fazem uso exclusivo da abordagem dominante do assunto. A questão central reside no adequado equilíbrio entre a pesquisa e o ensino nas Instituições e nos respectivos Cursos. A figura 1 (adaptada de MEYERSON, MASSY, 1994) ilustra de forma meridianamente clara esta questão.

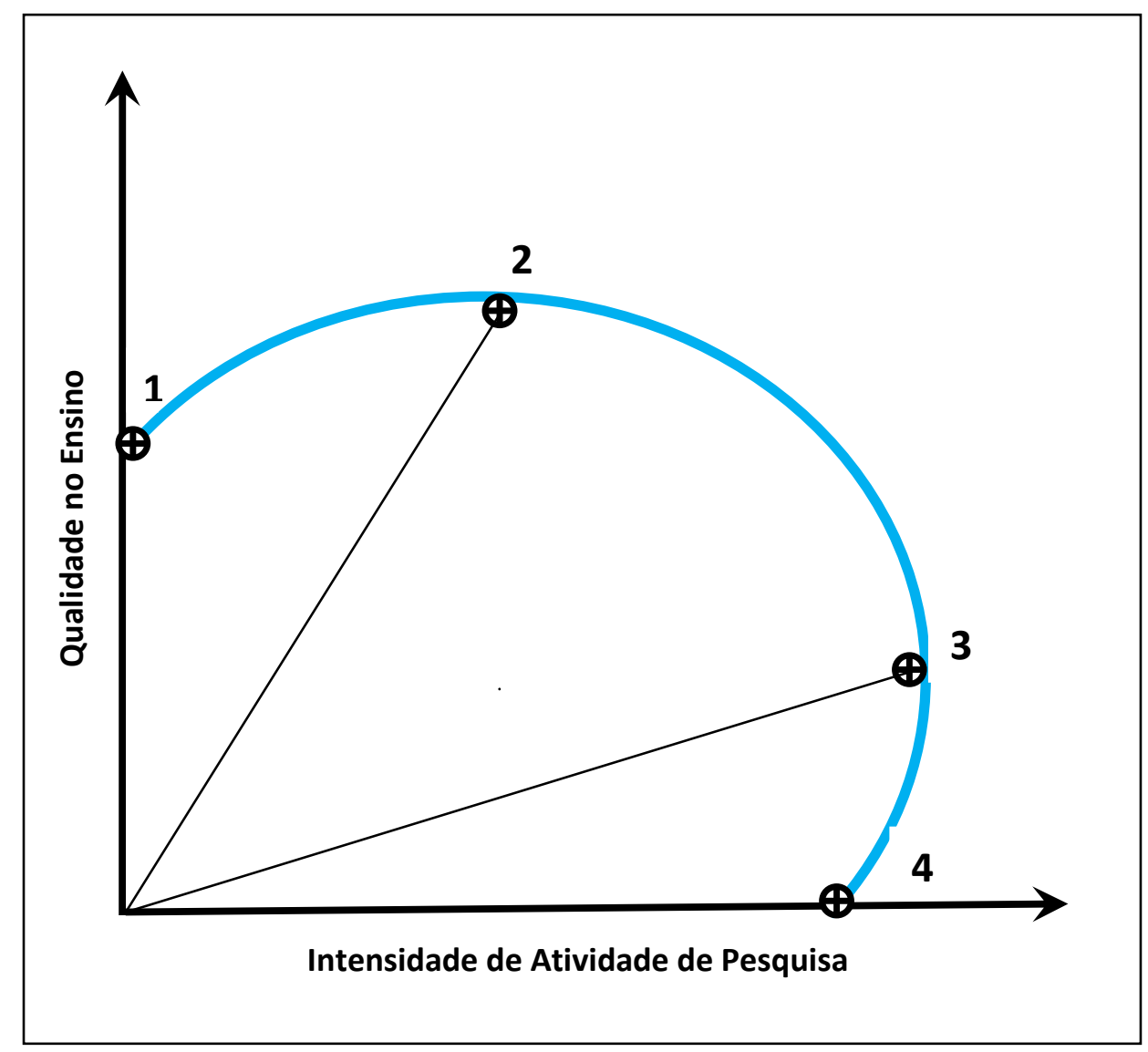

Figura 1 Pesquisa $x$ Ensino.

Fonte: Adaptada de MEYERSON, MASSY (1994).

Considerando-se uma quantidade fixa de recursos (humanos e materiais), as variáveis "intensidade de atividade de pesquisa" e "qualidade no ensino" se relacionam por meio da curva 1234. Ao longo do trecho $\mathbf{1} \rightarrow \mathbf{2}$ as variáveis são complementares, uma vez que a 
qualidade no ensino se beneficia do desenvolvimento de pesquisa. A partir do ponto $\mathbf{2}$, e no trecho $\mathbf{2} \rightarrow \mathbf{3}$, o crescimento da atividade de pesquisa implica em priorizar recursos humanos e materiais para a pesquisa em um nível tal, que impacta negativamente na sua função de ensino. Neste caso as componentes qualidade no ensino e intensidade de atividade de pesquisa não são complementares, mas sim, se substituem, uma vez que a quantidade de recursos é fixa. Incrementos na atividade de pesquisa a partir do ponto 2 demandam recursos em um patamar que prejudica o processo educacional. Neste trecho as variáveis são substitutivas. O ponto 4 representa a situação de uma instituição dedicada exclusivamente à pesquisa (instituto de pesquisa) cuja atuação será mais efetiva se dedicar uma parcela de sua energia na formação de recursos humanos. Esta situação é ilustrada na trajetória $\mathbf{4} \rightarrow \mathbf{3}$. Os descompromissados questionamentos e atitudes decorrentes da atividade educacional contribuem positivamente para oxigenar a atividade de pesquisa. Não é por outra razão que uma parcela expressiva dos institutos de pesquisa em funcionamento no Brasil fazem parte da estrutura de uma Instituição de Educação Superior ou oferecem cursos de pós-graduação Stricto Sensu. A intensificação da atividade educacional na trajetória $\mathbf{3} \rightarrow \mathbf{2}$, analogamente, implica em priorizar recursos para o ensino em detrimento da atividade de pesquisa.

Em síntese, a análise do vínculo entre a pesquisa e o ensino não pode ser reduzida a obtenção de uma única conclusão aplicável para toda e qualquer instituição de ensino. De fato, há situações onde o nível da atividade de pesquisa prejudica as tarefas de ensino. Nestes casos se atribuem elevados níveis de prioridade na aplicação de recursos para a agenda da pesquisa, e se delegam as atividades fundamentais do ensino para o corpo docente com baixa atividade em pesquisa Trata-se de uma divisão de trabalho que efetivamente não contribui para a Educação Superior (HATTIE, MARSH, 2004). Tipicamente este é o perfil das instituições funcionando no intervalo $\mathbf{2} \rightarrow \mathbf{3}$.

A relação entre ensino e pesquisa não segue um padrão único e homogêneo; ela é o resultado do que cada instituição atribui para cada uma delas. A despeito do discurso dos órgãos reguladores das Instituições de Educação Superior brasileiras manifestar a indissociabilidade entre as atividades de ensino e pesquisa, os incentivos governamentais explicitam que recursos se direcionam a atividades de pesquisa. Acrescenta-se, que os critérios para julgamento são norteados unicamente por resultados de atividades de pesquisa (NUNES, 2012). Em conclusão, a prática exercida no financiamento para a Educação Superior não contribui para associação entre ensino e pesquisa. 
Este trabalho tem como estratégia realizar de forma objetiva o acompanhamento das atividades de pesquisa de uma rede de ensino superior. Segundo Mukherji e Mukherji (2003) "tudo em estratégia é muito simples, mas não significa que seja muito fácil". Projetos gerais, mesmo que solidamente consistentes, não promovem as mudanças planejadas se não forem seguidos de um efetivo programa de acompanhamento e que, a partir dos resultados estimule efetuar correções de rumo. Para gestores que operam nos contextos em que a alocação de recursos está atrelada ao desempenho, é fundamental a disponibilidade de ferramentas que analisem objetivamente o desempenho (MILLER, 2006).

Projetos e programas episódicos não contribuem para o progresso de Instituições de Educação Superior. A liderança de uma Instituição de Ensino Superior deve focar sua atuação em um número relativamente reduzido de objetivos de elevada densidade acadêmica, a serem perseguidos obstinadamente por um intervalo de tempo que permita avaliar de fato seu impacto. Este trabalho descreve o procedimento atualmente em curso em uma rede de Educação Superior com ampla atuação no território brasileiro que coligiu durante cinco anos consecutivos um robusto conjunto de informações. De posse destes elementos são apresentados os procedimentos utilizados, análises dos resultados e medidas decorrentes.

\section{METODOLOGIA}

Programas de avaliação em Instituições de Ensino Superior devem abordar com rigor uma quantidade expressiva de enfoques da atividade acadêmica. A necessidade de se examinar vários parâmetros simultaneamente conduz gestores acadêmicos a adotar procedimentos excessivamente detalhados, em detrimento do objetivo final do processo. A eficácia do método está diretamente associada ao estabelecimento de um procedimento prático, e que priorize os aspectos mais relevantes. É, portanto, indispensável a aceitação com naturalidade, de que é possível que seja necessário descartar algum aspecto não central em beneficio do conjunto. No mundo acadêmico, por sua natureza, nem sempre este raciocínio prevalece e, com frequência não são absorvidas valiosas experiências que oferecem excelentes oportunidades de se alterar positivamente o status quo. O procedimento a ser utilizado deve necessariamente conduzir à execução de concretas medidas visando melhorias.

As Instituições de Ensino Superior estão progressivamente absorvendo uma cultura avaliativa, sem dúvida também induzidas por iniciativas dos órgãos reguladores. Os 
procedimentos avaliativos, a despeito de todas as dificuldades inerentes, facilitam a identificação de deficiências e o estabelecimento de objetivos e metas.

O acompanhamento de atividades de natureza acadêmica é sensivelmente mais complexo do que o de uma linha de produção e naturalmente menos suscetível à métrica. Nestas situações é frequente se observar tipicamente três possíveis atitudes;

a) julgamentos integralmente subjetivos e superficiais sob alegação da incomparabilidade dos desempenhos. Nestes casos passam a ser meramente opiniões ou dogmas (DAVENPORT, PRUSAK, 1998);

b) apuração do desempenho na pesquisa a partir de variáveis óbvias, mas insignificantes. Nestes casos não se obtém a informação necessária;

c) valorização do que é prontamente mensurável em detrimento da identificação da componente efetivamente relevante.

A subordinação ortodoxa à métrica distorce o processo por superproduzir o que se consegue medir, e negligenciar os aspectos relevantes por não serem mensuráveis. Este conjunto de dificuldades acrescenta um desafio adicional para se estabelecerem objetivos procedimentos de aferição da atividade de pesquisa, reconhecer as conquistas e orientar seu desenvolvimento.

No caso em estudo, optou-se por avaliar a produção intelectual dos docentes por considerar que esta reflete a atividade de pesquisa.

Os indicadores utilizados para avaliação da produtividade em pesquisa em questão foram constituídos em dois blocos:

- Produção bibliográfica com peso 3;

- Orientações concluídas com peso 1.

Dentro de cada bloco também foram aplicados diferentes pesos para cada tipo de produção cientifica que se encontram indicados no Quadro 1 a seguir. 


\begin{tabular}{|l|c|}
\hline \multicolumn{1}{|c|}{ Bloco/tipo de Produção } & Peso \\
\hline \multicolumn{1}{|c|}{ Produção bibliográfica } & $\mathbf{3}$ \\
\hline Artigos publicados em periódicos especializados (circulação nacional) & 35 \\
\hline Artigos publicados em periódicos especializados (circulação internacional) & 50 \\
\hline Trabalhos completos publicados em anais de eventos científicos, tecnológicos e artísticos & 15 \\
\hline Livros publicados & 30 \\
\hline Capítulos de livros publicados & 10 \\
\hline Resumos de trabalhos publicados em revistas técnico-científicas e periódicos especializados & 10 \\
\hline Resumos de trabalhos publicados em anais de eventos científicos, tecnológicos e artísticos & 5 \\
\hline \multicolumn{1}{|c|}{ Orientações concluídas } & $\mathbf{1}$ \\
\hline Teses & 50 \\
\hline Dissertações & 25 \\
\hline Trabalhos de conclusão de curso de graduação & 5 \\
\hline Iniciação científica & 5 \\
\hline
\end{tabular}

Quadro 1 Tipos de Produção e Peso.

O racional desenvolvido viabiliza mensurar a produção de cada docente por meio de um índice (IPP). A pontuação obtida por cada docente consiste na média ponderada dos tipos de produção normalizada pelo número de meses do período analisado. A produção científica de um docente é o reflexo da sua pesquisa, e que se manifesta em um intervalo de tempo compatível com a atividade desenvolvida. Para efeito deste trabalho considerou-se o registro da produção no período de três anos que, classicamente corresponde ao ciclo de avaliação dos órgãos reguladores.

Analisada a atividade de pesquisa do docente, é calculado o seu índice de produtividade. Em sequencia, se faz necessário situá-lo no curso e na instituição,

Objetivando avaliar a evolução da atividade de pesquisa no conjunto do curso e instituição se fez uso dos índices dos respectivos professores por intermédio de dois indicadores:

1. NEP (Nível de Envolvimento em Pesquisa), que indica o grau de disseminação da atividade de pesquisa no curso. Este indicador consiste no percentual de docentes que apresenta IPP diferente de zero sem apurar seu valor. Este parâmetro é um sinalizador do ambiente de pesquisa existente no Curso ou Instituição.

2. $\quad$ IPP $_{\text {Curso }}$ e IPP IES (Índice de Produtividade em Pesquisa do Curso e da Instituição, respectivamente), média do IPP de $25 \%$ dos docentes com maior pontuação. Na 
obtenção deste parâmetro não se aplica nenhum diferencial que incorpore a classificação da respectiva publicação. Esta metodologia, ainda que de forma simplificada, sinaliza com muita nitidez a existência e a intensidade de atividade de pesquisa.

A combinação destes dois indicadores fornece informações que possibilitam otimizar a aplicação de recursos para pesquisa identificando existência de grupos de pesquisadores com elevado potencial investigativo. Simultaneamente este mecanismo permite identificar cursos onde não se registra uma atividade de pesquisa mínima exigível para um curso.

A sistemática utilização destes indicadores ao longo de 05 anos consecutivos permitiu às IES envolvidas conhecerem, de forma tangível, seu desempenho na atividade de pesquisa, sua potencialidade, evolução no tempo e fragilidades. Objetiva-se com este procedimento obter "informações", que na definição de Peter Drucker consiste no fornecimento de dados relevantes com objetivo definido (DAVENPORT, PRUSAK, 1998).

\section{RESULTADOS}

A aplicação desta metodologia se realizou de forma gradual iniciando pelas IES de maior envergadura, e a cada ano incluindo novas instituições. Ao final de 2014 o sistema abrigava 35 IES com 8.892 professores atuando em 49 municípios e distribuídos em 82 campi.

Os resultados obtidos são agregados por IES, Centros de Conhecimento, Curso, Município e Campus. Semestralmente são elaborados relatórios densamente comentados contendo os resultados obtidos que são disponibilizados à totalidade da comunidade acadêmica e distribuídos para os respectivos gestores acadêmicos.

A estrutura organizacional acadêmica da rede de ensino analisada agrega 93 cursos de graduação distribuídos em sete áreas de conhecimento. As consolidações por IES e Centro de Conhecimento associam o docente uma única vez, enquanto que nos cursos, elas se realizam tantas vezes quantas os docentes atuem nos respectivos cursos. Dentro desta realidade, IPP $_{\text {Centro }}$ e IPP IES exibem a disponibilidade de docentes com atuação em pesquisa. O desdobramento deste índice por campus, curso e professor viabiliza aos Coordenadores de Curso, a identificação de talentos em pesquisa já existentes na Instituição. O procedimento adotado sinaliza objetivamente a vocação de seus docentes, e é particularmente eficaz em uma instituição multicampi com pluralidade na formação acadêmica de seus docentes. O simples conhecimento difuso da competência em pesquisa de seus docentes é de limitada utilidade. 
A Figura 2 ilustra a evolução semestral do IPP por cinco anos de toda a rede. A existência do índice, além de disponibilizar indicadores para gestão da produtividade científica, permite o acompanhamento de sua evolução no tempo.

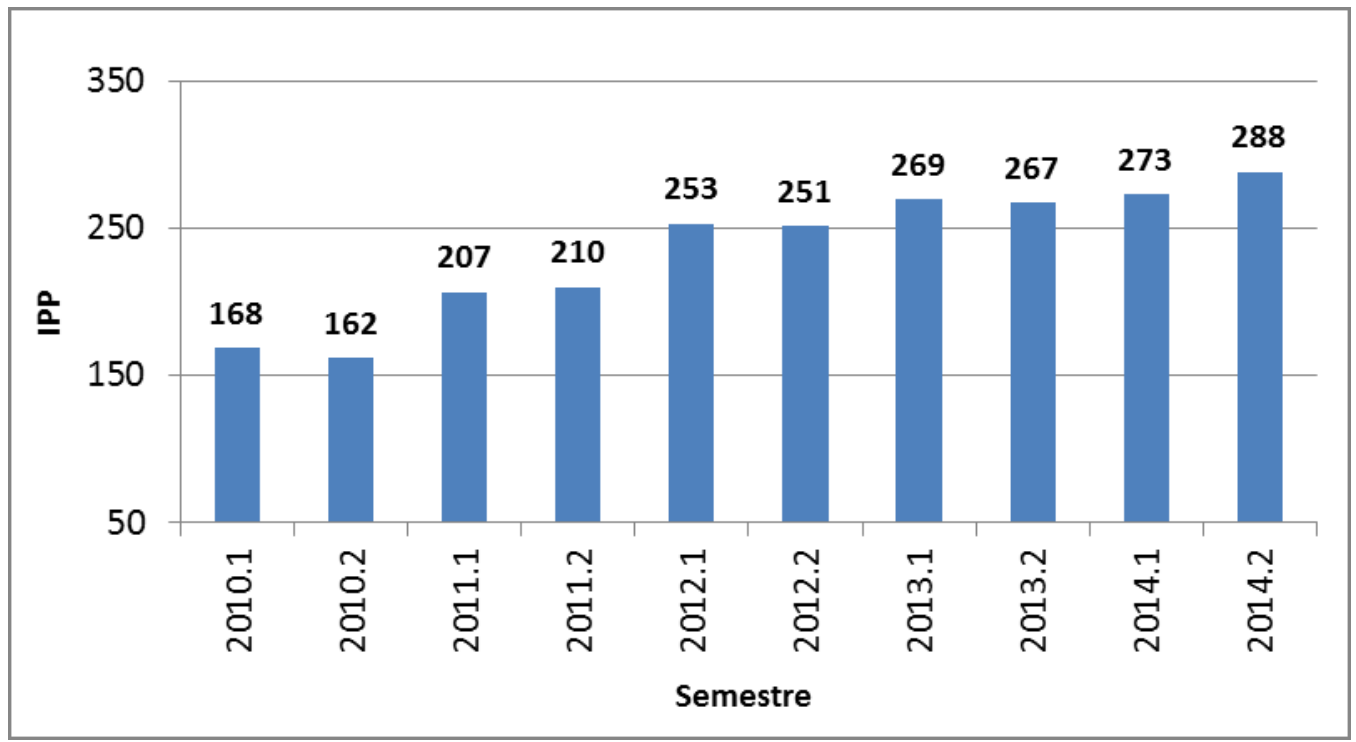

Figura 2 IPP $_{\text {Rede. }}$

Naturalmente as evoluções não se processam com a mesma intensidade entre todas as IES como ilustra a Figura 3 adiante. A IES de número 10 apresentou em 2011 um índice (154) substantivamente abaixo da média (210) e não registrou evolução positiva depois de

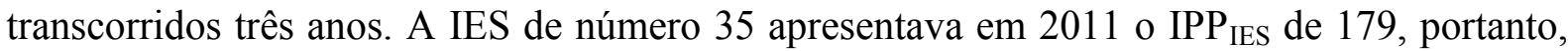
próxima da média e, após decorridos três anos, registrou um crescimento de $170 \%$. Esta métrica permite aos gestores institucionais reconhecerem os progressos existentes e identificar as IES com necessidade de ações para reversão de um quadro negativo. 


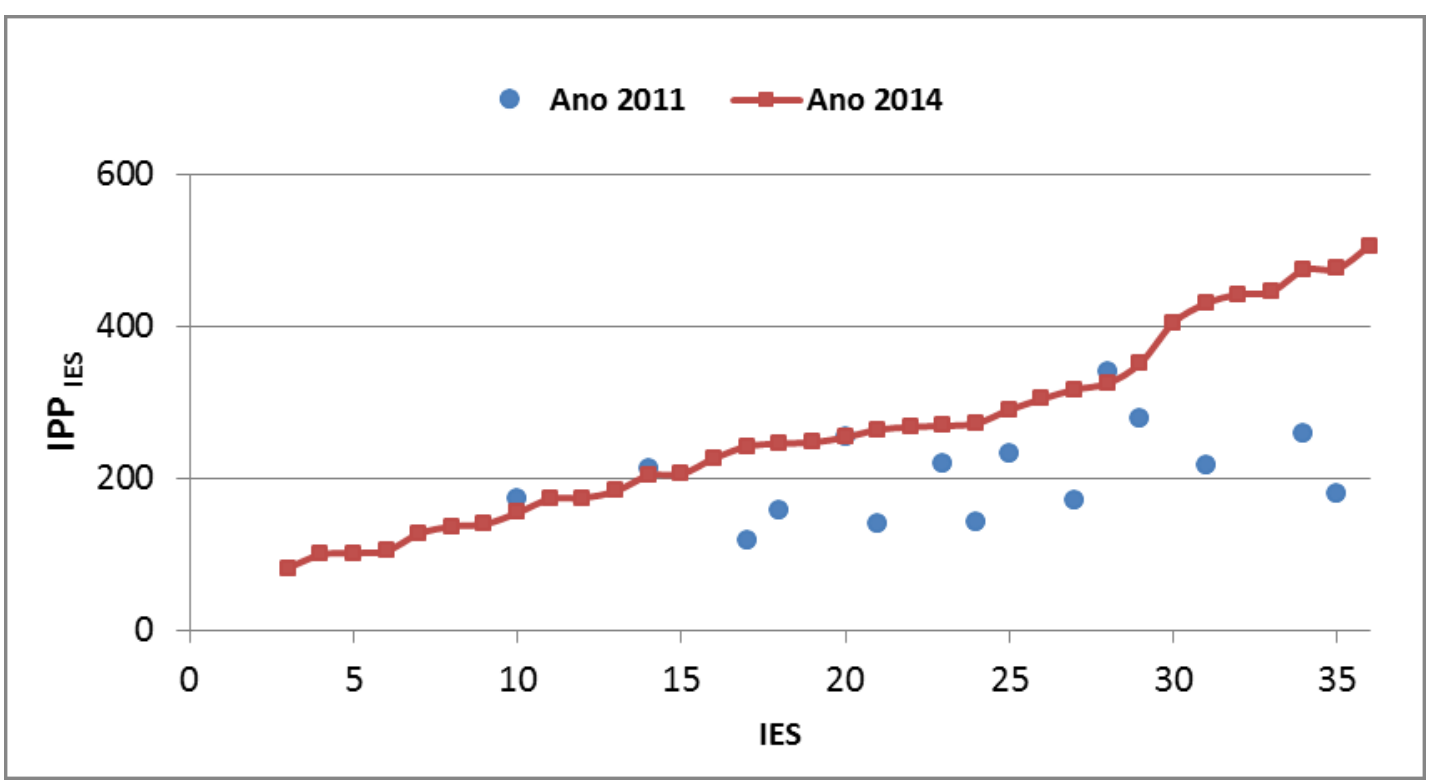

Figura 3 IPP por IES.

De forma semelhante, o gestor nacional de cada Centro de Conhecimento dispõe de informações relativas à sua respectiva área de atuação. A evolução entre os anos de 2010 e 2014 apresenta-se na Figura 4.

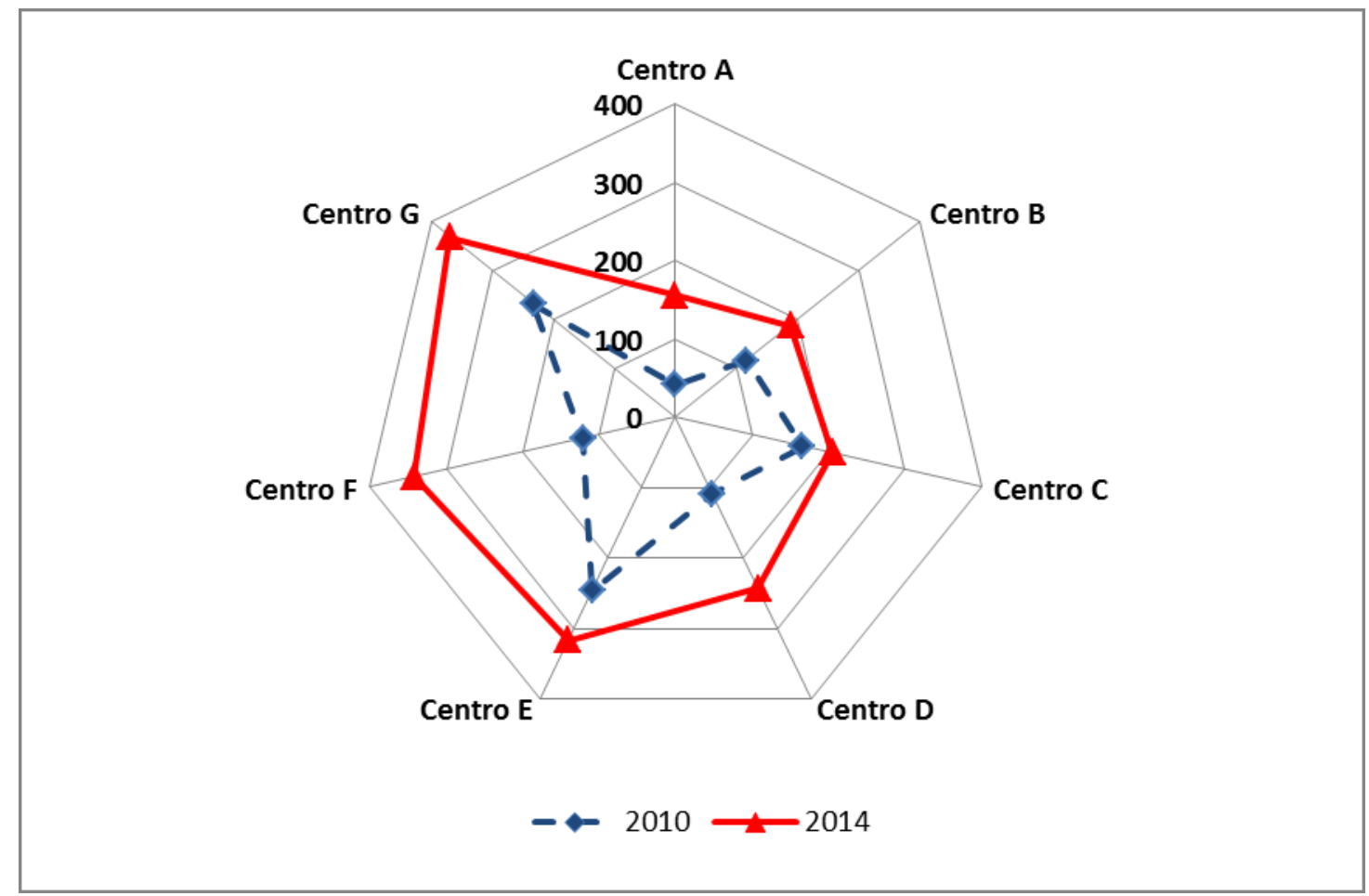

Figura 4 IPP por Centro de Conhecimento. 
A medida que se transita nos diferentes níveis de gestão se faz necessário maior detalhamento das informações. De forma semelhante, cada gestor de IES dispõe de informações sobre os cursos em funcionamento no seu Campus. Ao gestor de um Campus são disponibilizadas informações sobre os cursos em funcionamento onde também se identificam sensíveis diferenças, como indicado na Figura 5, que exibem simultaneamente $\operatorname{IPP}_{\text {Curso }}$ e o respectivo Nível de Envolvimento em Pesquisa (NEP). A situação ideal corresponde ao curso onde os dois parâmetros se encontram acima da média, revelando não só a existência de uma substantiva intensidade de pesquisa dos docentes mais ativos, bem como um percentual elevado de docentes com envolvimento em pesquisa, como se observa no curso 22 que possui

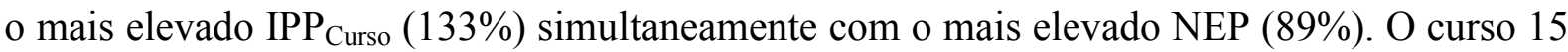

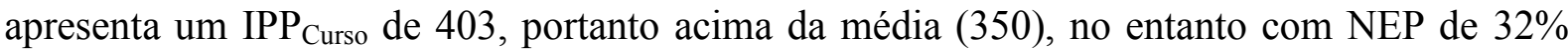
indicando que esta produção científica está concentrada em uma reduzida parcela de docentes.

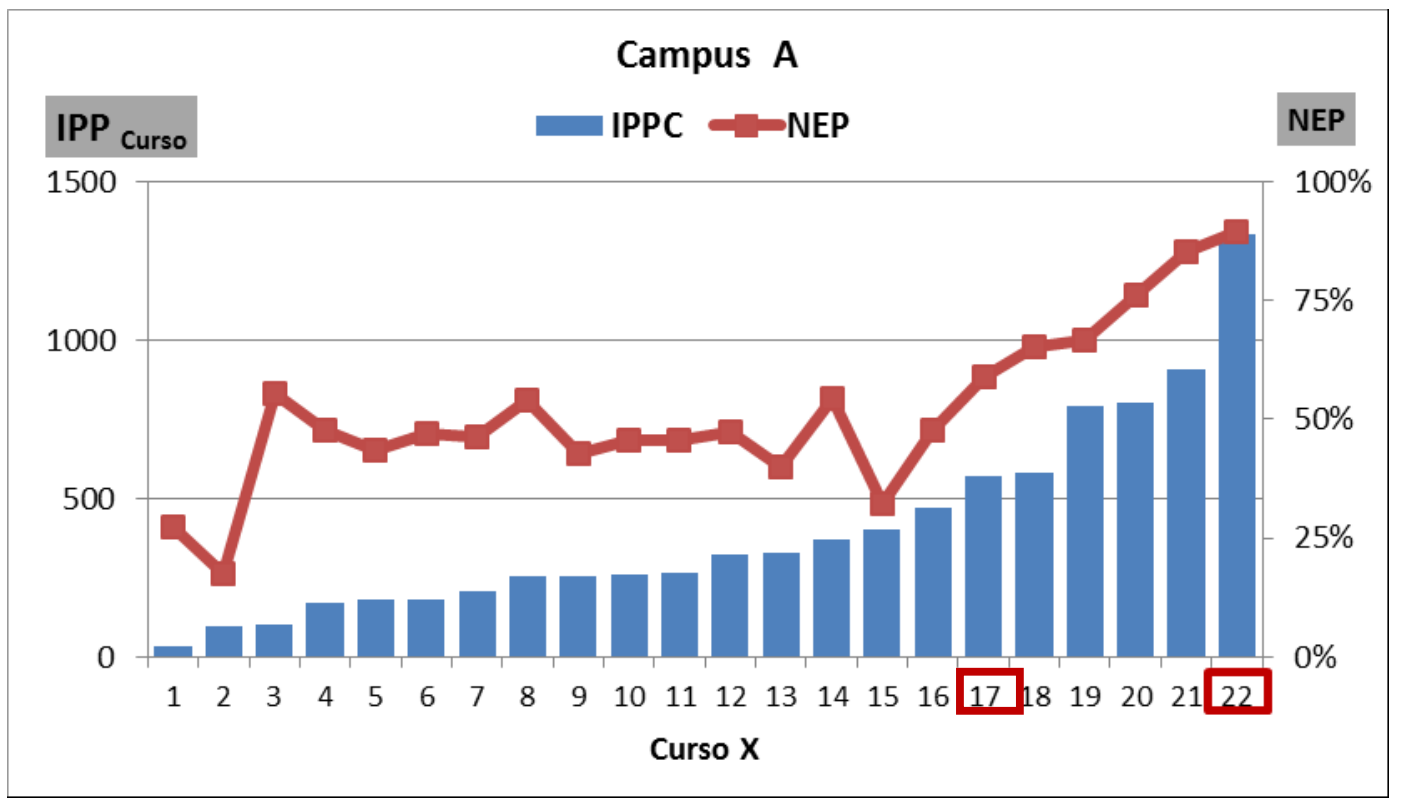

Figura 5 IPPC x NEP.

A visão transversal dos parâmetros nos respectivos municípios também possibilita identificar assimetrias dentro do mesmo curso em diferentes municipios. A Figura 6 a seguir revela que no curso em epígrafe se registra um $\operatorname{IPP}_{\text {Curso }}$ de 1.335 em um determinado

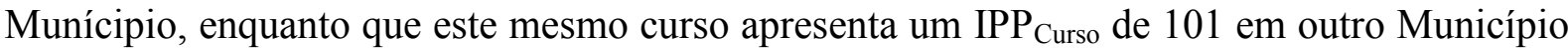
e que na realidade significa a inexistência de pesquisa. A atuação de ambos os cursos em uma mesma rede pode ser explorada para minimizar esta diferença uma vez que a atividade de pesquisa pode ser socializada entre docentes mais facilmente que o ensino, ainda que em 
diferentes IES. O procedimento preconizado para gestão de conhecimento neste trabalho fornece objetivas informações para tomada de decisão, entretanto, para dar consequência é necessário transformá-las em ações concretas. Dentro deste racional as coordenações de curso recebem IPP de cada um de seus docentes de forma a instrumentaliza-los a direcionar os estímulos à pesquisa institucional pela concessão de bolsas de produtividade em pesquisa e de iniciação científica, programas estes desenvolvidos pela IES.

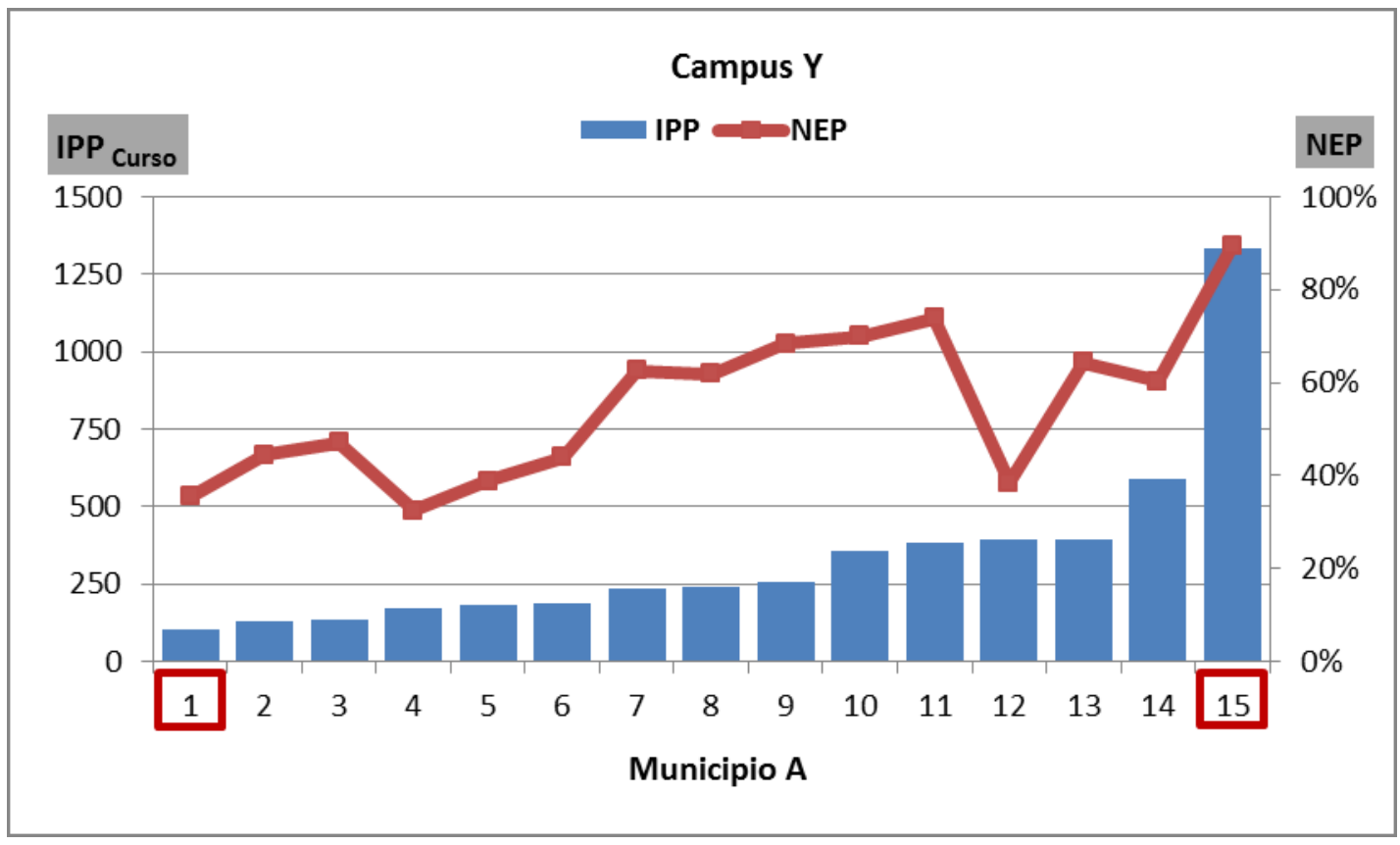

Figura 6 IPP e NEP no Campus.

Após cinco anos da utilização do presente mecanismo e seu contínuo acompanhamento, foram definidas metas de crescimento anual do IPP para docentes, Cursos, Áreas de Conhecimento e IES. A usual simplificação de se utilizar de uma meta única de crescimento para qualquer grandeza é no primeiro momento atraente, entretanto este procedimento não cumpre o papel reservado a adoção do sistema de metas. $\mathrm{O}$ estabelecimento de metas se destina a nortear e estimular o crescimento, porém, se aplicado sem compreender a dinâmica do processo, pode ter efeito contrário, ou por estabelecer valores inatingíveis, ou por facilitar seu atingimento sem esforço. Para valores iniciais do índice muito abaixo da média, é necessário o estabelecimento de uma agressiva meta de crescimento, enquanto que para valores do índice muito acima da média não é razoável se aplicar a mesma meta de crescimento. A partir da sensibilidade adquirida na apuração do IPP dos 8892 docentes em 
dez semestres consecutivos, estabeleceu-se a seguinte relação entre o crescimento do índice e seu valor presente.

META de CRESCIMENTO do IPP (\%) $=70000 /\left(\right.$ IPP) ${ }^{1,7}$

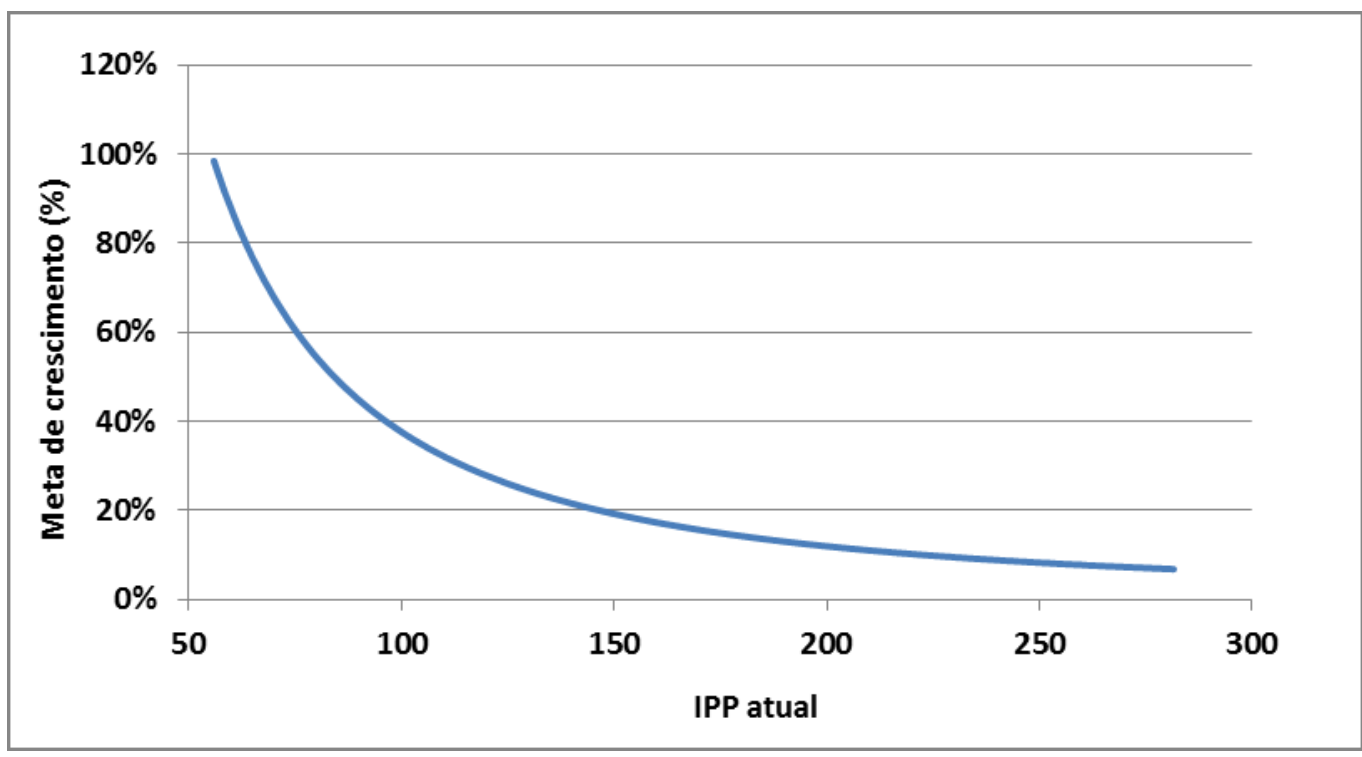

Figura 7 IPP x Meta.

O relatório, contendo resultados e análises semestrais, é acompanhado de metas a serem atingidas por cada docente, Curso e IES no ano subsequente. Faz parte dos relatórios semestrais a análise dos resultados cotejados com as metas estabelecidas no relatório anterior.

\section{CONCLUSÕES}

Um dos desafios que se apresentam para as Instituições de Ensino Superior consiste em sua necessidade de apurar o desenvolvimento resultante da pesquisa. A incessante busca de um abrangente modelo que considere todas as variáveis, inclusive aquelas com reduzida influência no resultado final, frequentemente abortam propostas utilizáveis em função da sua recusa em respeitar rigorosas premissas, nem sempre relevantes. $\mathrm{O}$ mito da incomparabilidade se sustentou durante muito tempo no meio universitário, entretanto, independentemente deste posicionamento, agências governamentais e a imprensa realizaram suas comparações a partir de informações fragmentadas e superficiais obtendo conclusões desprovidas de fundamentação. A avaliação do desempenho em pesquisa, ainda que com suas naturais dificuldades, deve ser realizada de maneira pratica e dela se extraírem soluções para resolver os problemas detectados. Estabelecer referência entre desempenhos em uma atividade se constitui em um processo sistemático de medir e comparar trabalho entre de indivíduos e 
organizações, trazendo uma visão externa, o que os autores deste trabalho consideram salutar. Dentre as diversas formas de tangibilizar a atividade de pesquisa de um docente, este trabalho optou por considerar a produção bibliográfica, atributo relevante, pois decorre de análise dos seus pares, e a orientação de alunos de graduação e pós-graduação stricto sensu. Neste contexto foi concebido um parâmetro, IPP, que pondera estas duas variáveis em ciclos de três anos e que é apurado para cada docente. Este parâmetro, individualizado por docente, é utilizado para consolidar a avaliação da atuação em pesquisa em um curso ou uma instituição por meio de dois indicadores, NEP (Nível de Envolvimento em Pesquisa) e IPP Curso $_{\text {IPP }}$ IES $_{\text {IE }}$ (Índice de Produtividade em Pesquisa do Curso ou Instituição).

A captura destas informações se realizou de forma automatizada ao longo de dez semestres consecutivos, a partir das informações disponíveis na Plataforma Lattes do CNPq via software customizado para a Instituição.

Esta metodologia foi divulgada e disseminada primordialmente no conjunto de docentes e gestores acadêmicos. Os resultados obtidos ao longo de cinco anos foram amplamente divulgados, acompanhados de análises semestrais e de metas a serem atingidas nos semestres subsequentes, e, cujo estabelecimento se realizou a partir dos desempenhos historicamente registrados levando em consideração o estágio em que cada docente e curso se encontram.

Este processo tangibilizou com transparência e universalidade o desempenho da atividade de pesquisa na rede de educação superior. Sua aplicação viabilizou identificar as competências instaladas em pesquisa, acompanhar as respectivas evoluções no tempo, estabelecer metas de melhoramento e priorizar tomadas de decisão.

A existência de um método para este fim e sua aplicação com regularidade estabeleceu uma cultura organizacional altamente favorável ao desenvolvimento da pesquisa. Esta metodologia que foi fundamentalmente direcionada aos gestores acadêmicos e docentes, dotou setores da rede estritamente gerenciais com indicadores que foram incorporados aos processos administrativos destinados a orientar a priorização de seus investimentos e reconhecer os talentos existentes. A partir da identificação de resultados objetivos decorrentes da aplicação desta metodologia, foram concebidos instrumentos internos de estimulo a atividade de pesquisa e mecanismos institucionais para incentivar a produtividade dos docentes na disputa por recursos externos voltados para pesquisa. Ambas as situações conduzem a disseminação e intensificação desta atividade por opção institucional. 


\section{REFERÊNCIAS BIBLIOGRÁFICAS}

CESAR, S.B.; MUYLDER, C.F e MARTINS, H.C. (2014) A gestão do conhecimento na indissociabilidade Ensino, Pesquisa, Extensão. In: $12^{\circ}$ CONGRESSO BRASILEIRO DE GESTÃO DO CONHECIMENTO- KM BRASIL, 2014, Florianópolis. Disponível em: $<$ http://www.kmbrasil.com/anais/arquivos/trabalhos/48.pdf $>$. Acesso em 23 abril 2015.

CLARK, B.R. On Higher Education: Selected Writings 1956-2006. Baltimore: Johns Hopkins Press, 2008.

DAVENPORT, T.; PRUSAK, L. Working Knowledge: How organizations Manage they Know. Boston: Harvard Business School Press, 1998.]

FARIA, R.M. Ciência Tecnologia e Inovação para um Brasil Competitivo. São Paulo: SBPC, 2011.

GESCHWIND, L.; BROSTREOM, A. Managing the teaching-research nexus: ideals and practice in research oriented universities. Higher Education Research \& Development, Auckland, v. 34, n. 1, p. 60-73, 2015.

HATTIE, J.; MARSH, H.W. One journey to unravel the relationship between research and teaching. Apresentado em: Research and Teaching: Closing the divide? An International Colloquium, 2004. Winchester. Disponível em: <

https://cdn.auckland.ac.nz/assets/education/hattie/docs/relationship-between-research-andteaching-\%282004\%29.pdf $>$. Acesso em 23 abril 2015.

MEYERSON, J.W.; MASSY, W. F. Measuring institutional performance in higher education. Princeton: Peterson's, 1994.

MILLER, B.A. Assessing organizational performance in higher education. San Francisco: Jossey- Bass, 2006.

MUKHERJI, A.; MUKHERJI, J. (2003) Understanding strategy; why is strategy so difficult? Advanced in Competitiveness Research. Indiana, v. 11, n. 1, p 1-19, 2003.

NUNES, E.O. Educação Superior no Brasil: Estudos, debates, controvérsias. Rio de Janeiro: Garamond Universitária, 2012. 